\title{
Automatic flow systems based on sequential injection analysis for routine determinations in wines
}

\author{
Marcela A. Segundo $^{a}$,*, José L.F.C. Lima ${ }^{a}$, António O.S.S. Rangel ${ }^{b}$ \\ ${ }^{a}$ REQUIMTE, Departamento de Química-Física, Faculdade de Farmácia, Universidade do Porto, R. Aníbal Cunha, 164, 4050-047 Porto, Portugal \\ ${ }^{\mathrm{b}}$ Escola Superior de Biotecnologia, Universidade Católica Portuguesa, R. Dr. António Bernardino de Almeida, 4200-072 Porto, Portugal
}

Keywords: Wine; Sequential injection analysis; In-line sample treatment

\begin{abstract}
Sequential injection systems for wine analysis have been developed in recent years for determination of more than 20 species. Several aspects of these systems are reviewed in the present paper. Special focus is given to implementation of in-line sample treatment and adaptation of system operation through software control to enable determination in different types of wine. The strategies used to enhance selectivity and the capacity for multi-parameter determination are also addressed.
\end{abstract}

\section{Introduction}

Quality control is an essential requirement of modern wine making in order to have the most efficient use of the available resources to produce wines of an agreed standard. This can only be possible by having enough information about the composition of wine during production and also in the final product. This situation requires an enormous number of chemical analyses in order to determine ethanol, sugars, organic acids, sulphite, minerals and other relevant compounds in wine. The demand to carry out a large number of assays routinely justifies the introduction of automatic methods.

Systems for automation of wine analysis based on flow techniques have been described and applied over the past years. In fact, analysers based on segmented flow analysis (SFA) had been very successful, with recognised efficiency [1]. Nevertheless, they presented the disadvantages inherent to this type of system, where measurements rely on physical and chemical equilibrium conditions. For instance, a long start-up time is required before operation and many samples and reagents can be lost if the system function is interrupted abruptly.

* Corresponding author. Fax: +351-222004427.

E-mail address: msegundo@mail.ff.up.pt (M.A. Segundo).
Automatic systems based on flow injection analysis (FIA) were also described for wine analysis, but they did not have the same implementation as SFA systems. In a comprehensive review of FIA systems for wine analysis, Ferreira et al. [2] pointed out that the systems developed until then had some limitations as many were only tested with a certain wine type or demanded a previous treatment of the sample before injection. The lack of robustness of some manifold components (tubing of peristaltic pumps, some types of injection devices) was also indicated as a possible reason for not using FIA in an industrial environment [3].

Sequential injection analysis (SIA), described as a mechanically simpler alternative to FIA [4], is a feasible choice for automation of wine analysis. In its simplest configuration, SIA consists in the sequential aspiration of well-defined sample and reagent zones into a holding coil by means of a multi-position valve. The flow is then reversed and the stacked zones are mixed and propelled to the detector, where the reaction product formed is monitored (Fig. 1). All operations are coordinated through computer control, including port selection in the multi-position valve and pump activation (flow rate and direction, operation time). Moreover, data acquisition is possible if the detection system is also connected to the computer.

Although SIA was first conceived as a single pump, single valve, single channel technique, the developed applications 


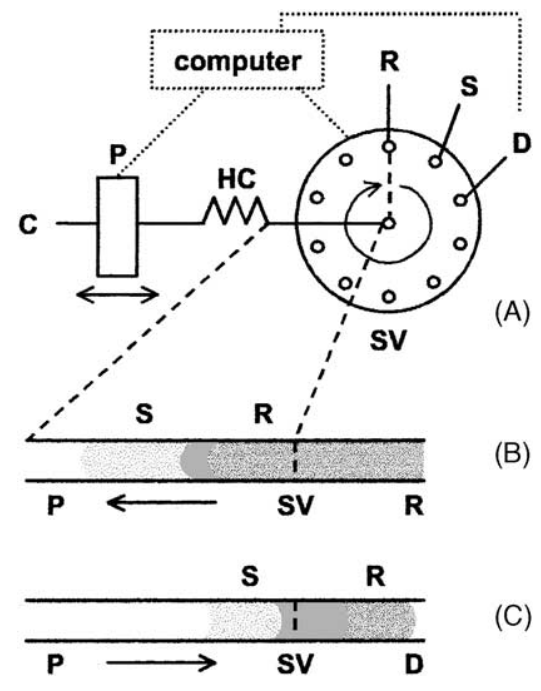

Fig. 1. Schematic representation of a SIA system (A). The holding coil zone is presented in more detail during the operational cycle, where sample and reagent are sequentially aspirated (B) and sent towards the detector after flow reversal (C). P, pump; SV, selection valve; C, carrier solution; HC, holding coil; D, detector; R, reagent; S, sample.

until now, recently reviewed by Lenehan et al. [5], showed that it can be used for more complex operations than simple addition of reagents to a sample. As different devices can be clustered around the selection valve, in-line sample treatment (gas diffusion, dialysis, digestion, extraction) can easily be accomplished. All these features were explored in SIA systems developed for wine analysis, which are discussed in this paper. The strategies used to enhance selectivity and the capacity for multi-parameter determination are also addressed.

\section{Application of SIA to wine analysis}

In wine analysis several aspects must be considered, namely the diversity of analytes, the complexity of the wine matrix and the different concentration ranges in which analytes can be found, depending on the type of wine. Therefore, the analytical method should be based on a reaction or detection system that is selective to the target analyte. When this situation is not possible, efficient removal or minimisation of matrix interference must be accomplished, preferably using in-line sample treatment. Further, as concentration values of a certain analyte can be rather different depending on the type of wine, determination over a wide concentration range or the possibility of adapting the working conditions to this situation are also important.

Several of these specifications were included in SI systems developed for wine analysis. As listed in Table 1, 15 manifolds have been described, allowing the determination of different parameters. This information was gathered from search engines available through the ISI Web of Knowledge. In these manifolds, different types of detection systems were employed, using spectrophotometry [6-13], amperometry $[14,15]$, potentiometry $[16,17]$, flame atomic absorption spectrometry (FAAS) $[18,19]$ and Fourier transform infrared (FTIR) spectrometry [20]. Most of the manifolds included or required some sort of sample treatment before reaction and/or detection. In-line sample treatment was implemented by connecting different devices to the lateral ports of the selection valve, as depicted schematically in Fig. 2. In the following sections, some features of these SIA systems are discussed, including their application to different types of wine samples and the strategies adopted to circumvent matrix interference.

\section{In-line sample treatment}

\section{Dilution and $\mathrm{pH}$ adjustment}

In most analytical methods, concentrations found in samples are above the range in which determination can be carried out. This can be easily overcome by diluting the sample. A similar situation is found for $\mathrm{pH}$ values, which can be adjusted by addition of buffer solution or concentrated alkaline or acid solutions. These operations were carried out in-line in SIA systems by means of a mixing chamber placed in a lateral port of the selection valve.

In the system described by Schindler et al. [20] the mixing chamber was used not only to dilute but also to acidify the sample. In this case, simultaneous determination of nine different wine components was based on multivariate evaluation of mid-IR transmission spectra of wine samples. As the IR spectra of protonated and deprotonated organic acids differ greatly, $\mathrm{pH}$ adjustment to values between 1.5 and 2.0 was required. Hence, all the organic acids were in the protonated form, reducing the number of species with different spectra. Results obtained for five wine samples were similar to those attained by a conventional liquid chromatography (LC) method.

A mixing chamber was used with the same purpose in the SIA system for determination of sulphate [13]. The assay was based on precipitation of $\mathrm{BaSO}_{4}$, measured turbidimetrically. As sample dilution and acidification were required, both sample and acid were sent to the mixing chamber; after thorough mixing, up to six aliquots could be drawn and analysed. This feature enhanced sample throughput by excluding sample preparation and chamber washing after each determination. Moreover, blank measurements were carried out from the same sample preparation. Ten wine samples (both red and white) were analysed, giving values similar to those provided by the gravimetric batch procedure.

\section{In-line dialysis and gas diffusion}

Wine colour, especially in red wines, can be a serious interference where spectrophotometric determination is concerned. In order to minimise the effect, SIA systems incorporating membrane separation units to allow in-line sample treatment have been described (Fig. 3). 
Table 1

SIA systems for wine analysis

\begin{tabular}{|c|c|c|c|c|c|c|c|}
\hline Analyte & $\begin{array}{l}\text { Detection } \\
\text { system }\end{array}$ & Application range & Sample pre-treatment & Sample in-line treatment & $\begin{array}{l}\text { Sampling } \\
\text { rate }\left(\mathrm{h}^{-1}\right)\end{array}$ & $\begin{array}{l}\text { RSD } \\
(\%)\end{array}$ & Reference \\
\hline Acetic acid & FTIR & $0.15-0.60 \mathrm{~g} \mathrm{l}^{-1}$ & - & Dilution and acidification & 20 & $<30$ & [20] \\
\hline Amino acids & UV/Vis & $28-140 \mathrm{mg}(\mathrm{N}) 1^{-1}$ & Filtration and dilution $(1: 1)$ & - & 12 & $<1.5$ & [11] \\
\hline Citric acid & FTIR & $0.3-1.6 \mathrm{~g} \mathrm{l}^{-1}$ & - & Dilution and acidification & 20 & $<8$ & [20] \\
\hline \multirow{2}{*}{ Copper } & FAAS & $0.005-2.00 \mathrm{mg} \mathrm{l}^{-1}$ & - & - & 75 & $<3$ & [18] \\
\hline & & $0.001-0.50 \mathrm{mg}^{-1}$ & - & Solid phase extraction & 20 & $<3$ & \\
\hline \multirow[t]{4}{*}{ Ethanol } & Amperometry & $0.17-1.71 \mathrm{mmol}^{-1}$ & Dilution (1:1000-1:1667) & - & - & $<2.9$ & [14] \\
\hline & Amperometry & $1-250 \mu \mathrm{moll}^{-1}$ & - & Dilution & - & $<2.7$ & [15] \\
\hline & FTIR & $79-91 \mathrm{~g} \mathrm{l}^{-1}$ & - & Dilution and acidification & 20 & $<2$ & [20] \\
\hline & $\mathrm{UV} / \mathrm{Vis}$ & $0.10-0.50 \%(\mathrm{v} / \mathrm{v})$ & Dilution (1:50) & - & 22.5 & $<3.4$ & [12] \\
\hline Fructose & FTIR & $1.5-7.5 \mathrm{~g} \mathrm{l}^{-1}$ & - & Dilution and acidification & 20 & $<8$ & [20] \\
\hline \multirow[t]{2}{*}{ Glucose } & Amperometry & $0.01-1.50 \mathrm{mmol}^{-1}$ & Dilution (1:3.3-1:30) & - & - & $<2.9$ & [14] \\
\hline & FTIR & $0.4-6.9 \mathrm{~g} \mathrm{l}^{-1}$ & - & Dilution and acidification & 20 & $<8$ & [20] \\
\hline \multirow[t]{2}{*}{ Glycerol } & FTIR & $5.9-8.1 \mathrm{~g} \mathrm{l}^{-1}$ & - & Dilution and acidification & 20 & $<8$ & [20] \\
\hline & UV/Vis & $0.03-0.30 \mathrm{~g} \mathrm{l}^{-1}$ & Dilution (1:50) & - & 22.5 & $<1.6$ & [12] \\
\hline $\begin{array}{l}\text { Glycerol and } \\
\text { 2,3-butanediol }\end{array}$ & Potentiometry & $2.5-20 \mathrm{~g}^{-1}$ & - & Ion exchange column & 33 & $<1$ & {$[16]$} \\
\hline Iron(III) & FAAS & $0.10-6.00 \mathrm{mg} \mathrm{l}^{-1}$ & - & Liquid-liquid extraction & 18 & $<4.9$ & [19] \\
\hline \multirow[t]{7}{*}{ Iron } & FAAS & $0.47-15.00 \mathrm{mg} \mathrm{l}^{-1}$ & - & - & 109 & $<3$ & [18] \\
\hline & & $0.20-10.00 \mathrm{mg} \mathrm{l}^{-1}$ & - & - & 109 & $<3$ & \\
\hline & & $0.10-5.00 \mathrm{mg} \mathrm{l}^{-1}$ & - & - & 95 & $<3$ & \\
\hline & UV/Vis & $2-14 \mathrm{mg} \mathrm{l}^{-1}$ & - & Microwave digestion & 10 & $<2.3$ & [9] \\
\hline & FAAS & $1.50-15.00 \mathrm{mg} \mathrm{l}^{-1}$ & - & - & 18 & $<1.9$ & [19] \\
\hline & & $0.50-10.00 \mathrm{mg} \mathrm{l}^{-1}$ & - & - & 18 & $<1.9$ & \\
\hline & & $0.25-5.00 \mathrm{mg} \mathrm{l}^{-1}$ & - & - & 18 & $<1.9$ & \\
\hline \multirow[t]{2}{*}{ Lactic acid } & UV/Vis & $0.25-2.5 \mathrm{~g}^{-1}$ & $\begin{array}{l}\mathrm{pH} \text { adjustment to } 13 \\
\text { with } 5 \mathrm{moll}^{-1} \mathrm{NaOH}\end{array}$ & Dialysis & 14 & $<2$ & [6] \\
\hline & FTIR & $0.4-2.8 \mathrm{~g} \mathrm{l}^{-1}$ & - & Dilution and acidification & 20 & $<8$ & [20] \\
\hline Malic acid & FTIR & $0.02-3.2 \mathrm{~g}^{-1}$ & - & Dilution and acidification & 20 & $<8$ & [20] \\
\hline Manganese & FAAS & $0.007-3.00 \mathrm{mg} \mathrm{l}^{-1}$ & - & - & 95 & $<3$ & [18] \\
\hline Phosphorus (total) & UV/Vis & $20.0-400.0 \mathrm{mg} \mathrm{l}^{-1}$ & - & Microwave digestion & 16 & $<3$ & [7] \\
\hline Potassium (free) & Potentiometry & Not given & - & - & 14 & $<3$ & [17] \\
\hline Potassium (total) & Potentiometry & Not given & - & Microwave digestion & 14 & $<3$ & [17] \\
\hline \multirow[t]{2}{*}{ Reducing sugars } & $\mathrm{UV} / \mathrm{Vis}$ & $2-25 \mathrm{gl}^{-1}$ & - & Dialysis & 14 & $<2.1$ & [8] \\
\hline & & $20-140 \mathrm{gl}^{-1}$ & & & 18 & & \\
\hline Sulphate & Turbidimetry & $300-1500 \mathrm{mg} \mathrm{l}^{-1}$ & - & Dilution and acidification & 5 & $<10$ & [13] \\
\hline Sulphur dioxide (free) & UV/Vis & $2-40 \mathrm{mg}^{-1}$ & - & Gas diffusion & 17 & $<1.2$ & [10] \\
\hline Sulphur dioxide (total) & UV/Vis & $25-250 \mathrm{mg} \mathrm{l}^{-1}$ & - & Digestion and gas diffusion & 16 & $<2.3$ & {$[10]$} \\
\hline Tartaric acid & FTIR & $1.0-1.7 \mathrm{~g} \mathrm{l}^{-1}$ & - & Dilution and acidification & 20 & $<8$ & [20] \\
\hline Zinc & FAAS & $0.005-2.00 \mathrm{mg} \mathrm{l}^{-1}$ & - & - & 95 & $<3$ & {$[18]$} \\
\hline
\end{tabular}

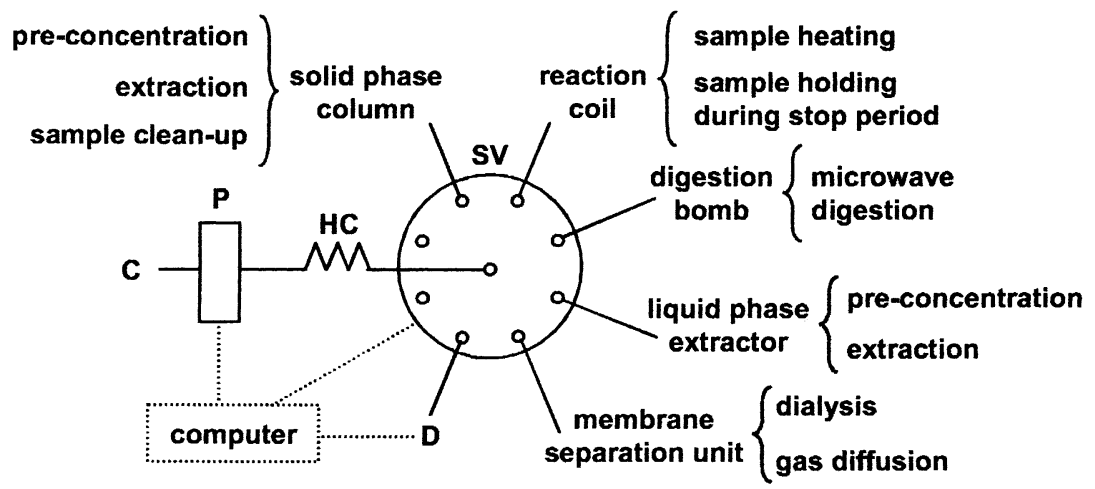

Fig. 2. Schematic representation of a SIA system where different devices are clustered around the selection valve. The operations performed for in-line wine sample treatment are indicated for each device. P, pump; SV, selection valve; C, carrier solution; HC, holding coil; D, detector. 

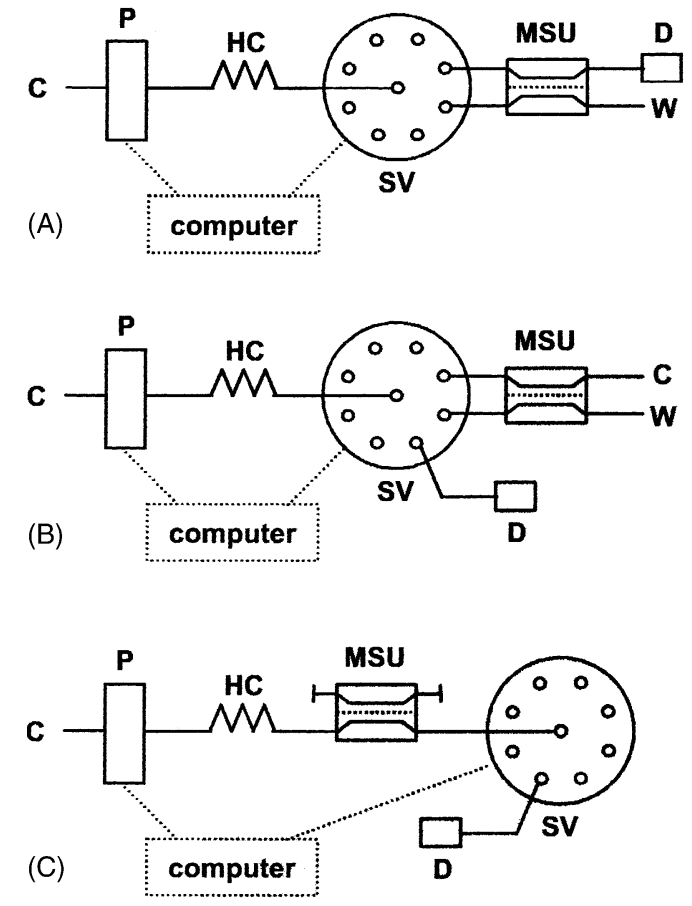

Fig. 3. Schematic representation of SIA systems incorporating membrane separation units (MSU) for in-line sample treatment. Different configurations are described for determination of sulphur dioxide $(\mathrm{A})$, reducing sugars (B) and L(+)lactate (C). P, pump; SV, selection valve; C, carrier solution; HC, holding coil; $\mathrm{D}$, detector; W, waste.

Free and total sulphur dioxide were determined by reaction with pararosaniline and formaldehyde, after passing through a gas diffusion membrane [10]. In the described manifold, each channel of the gas diffusion unit was connected to a different port of the selection valve (Fig. 3A). This configuration allowed discontinuous operation of donor and acceptor channels. Hence, lower reagent consumption was achieved as only the necessary amount was drawn into the holding coil and then sent to the acceptor channel. Further, it was possible to send different sample volumes through the donor channel while the acceptor line (reagent) was static. Consequently, determination in different concentration ranges was performed without physical reconfiguration of the manifold, according to the sample volume chosen in the controlling software. Ten wine samples (red or white) were analysed; no systematic differences were found between the results obtained by this method and by the titrimetric Ripper method.

A similar strategy was implemented in the system described for determination of reducing sugars [8]. These species were determined after passage across a dialysis membrane (Fig. 3B); this step was performed not only to minimise sample colour interference in the detection system, but also to dilute the sample. Different sample volumes were sent through the donor channel while the acceptor stream remained static. Afterwards, the acceptor plug was drawn into the holding coil and sent along with the reagent into a reaction coil. There, reduction of $\mathrm{Cu}$ (II) took place by reducing sugars, and the $\mathrm{Cu}(\mathrm{I})$ formed was detected as a complex with neocuproine. Different concentration ranges were attained through software control of operational conditions (sample volume, flow rate of donor stream). This enabled direct introduction of wine samples with low (table wine) or high (Port wine) sugar content. Nineteen samples were analysed and the results obtained were similar to those given by the titrimetric reference procedure.

A dialysis unit was also included in a SIA system to determine $\mathrm{L}(+)$-lactate in wines using enzymes (lactate oxidase and peroxidase) and spectrophotometric detection [6]. In this case, one channel of the dialysis unit was connected to the flow system, placed between the selection valve and the pump, while the other channel was closed, filled with carrier solution (Fig. 3C). Using this configuration, the wine sample was aspirated through the open channel and analyte was transferred to the static channel. After a stop period, the open channel was flushed to remove sample residues. Finally, reagent was aspirated through the open channel while analyte diffused back to this channel, where colour development took place. This approach minimised interference from the sample matrix and allowed high sample dilution. Eight samples (red and white table wines) were analysed after $\mathrm{pH}$ adjustment outside the manifold; the results were in good agreement with those provided by the batch method using $\mathrm{L}(+)$-lactate dehydrogenase and UV detection.

\section{In-line extraction}

Extraction procedures are often carried out in analytical methods. There are two main objectives: removal of analyte from interfering matrix or analyte enrichment in order to achieve determination at low concentrations. This type of procedure was implemented in-line in two SIA systems for wine analysis.

In the system described for determination of trace levels of copper, solid phase extraction was performed before FAAS detection [18]. In this procedure, diethylammonium- $N, N$ diethyldithiocarbamate (DDTC) was added in a confluence to the wine sample, before passing through a cartridge packed with $\mathrm{C} 18$ silica, where the $\mathrm{Cu}$ chelate was retained. Subsequently, it was eluted with ethanol and sent to the detection system. About 20 samples (different wine types: Verde, Maduro and Port) were analysed; the results obtained were in good agreement with those provided by the official Portuguese standard addition method.

In-line extraction was also performed in the SIA system for speciation analysis of iron in wines, using only one detection device [19]. Total iron was determined by sending a small sample aliquot directly to the FAAS detector; the determination of iron(III) was carried out after liquid-liquid extraction of the complex formed between iron(III) and thiocyanate, using methyl-iso-butylketone as the organic phase. In-line extraction was carried out in a device connected to a lateral port of the selection valve. This device consisted of a glass vial with a porous ceramic plate inside it which allowed solvent less dense than water to float above it. 
Hence, the extraction procedure was carried out in this organic phase by passing a mixture of sample and thiocyanate downward through it. After that, the content of the vial glass was washed with water to remove sample residues. Finally, the organic phase was aspirated back to the holding coil and then propelled to the detector. Red and white wine samples ( $n=10)$ were analysed by the described method and the results were compared to those obtained by the colorimetric thiocyanate method for $\mathrm{Fe}(\mathrm{III})$ and the FAAS method for total Fe. The absence of significant differences between the two sets of results was confirmed statistically.

\section{Microwave digestion}

Digestion procedures are usually required when determination of all forms of a certain species is intended especially in complex matrices. SIA can easily accommodate in-line sample digestion by placing a proper device in one of the lateral ports of the selection valve. Using this configuration, three different manifolds were developed to perform determination of total species with spectrophotometric detection and in-line microwave digestion.

The spectrophotometric determination of total phosphorous as phosphate ion, based on the formation of molybdenum blue, was described by Oliveira et al. [7]. In this case, the sample was transported together with nitric acid to a digestion bomb placed inside a microwave oven for subsequent digestion. The sample zone was stopped inside the oven and, after digestion, reagents were added to it. After that, the digestion bomb contents were directed in reverse flow into the holding coil and then sent to the detector. Using this operation cycle, the digestion bomb also acted as a mixing device promoting homogenisation regardless the number of reagents required. Moreover, the air bubbles formed during digestion were efficiently disposed of. As this application was focused on several different food samples, only two wine samples (red and white) were analysed; recovery studies were performed but no comparison with reference procedures was established.

The determination of total iron as iron(II) was performed in white wines after microwave digestion using a SIA system and spectrophotometric detection [9]. Colour development, using 1,10-phenanthroline and ascorbic acid, was implemented using an operational cycle similar to that described above. The system was applied to white wine samples (number not given); it was not suitable for red wines due to the spectral overlap of this type of wine and the coloured complex formed. A recovery experiment was described to assess accuracy, but no comparison with reference procedures was performed.

In the SIA system proposed by Zárate et al. [17], it was possible to carry out measurements of free and total potassium with a single detection system. Free potassium content was determined by aspirating a volume of sample followed by its propulsion to the potentiometric detector. Total potassium content was assayed using the same detector after sample digestion in a microwave oven. The digestion step included addition of hydrogen peroxide and it took place in a digestion bomb connected to one of the lateral ports of the selection valve. Ten wine samples (red, white and Port) were analysed. Results obtained for total potassium content were compared with those provided by flame emission photometry, which were statistically similar. For the determination of free potassium, recovery studies for three wine samples were performed to evaluate the accuracy and possible matrix interference.

\section{Miscellaneous}

Besides those already described, other strategies were devised to minimise matrix interference as in the SIA system for the determination of glycerol and 2,3-butanediol in wines [16]. The method was based on the reaction of these analytes with periodate, which was monitored by means of a periodate-selective electrode. Although potentiometric detection is considered a wise choice when sample colour is an issue, the methodology chosen suffered interference from other reducing components (mannitol and sorbitol) present in the wine matrix. In order to minimise this effect, a mini-column packed with anionic resin was coupled to a lateral port of the valve. Samples passed through it before reaction and detection. Recovery studies were performed using nine wine samples (different types) but no comparison to reference procedures was reported.

Matrix interference was also circumvented in the spectrophotometric SIA system described for the determination of primary amino acid concentration during wine fermentation [11]. In this case, the method was based on the formation of isoindole derivatives from the primary amino acid by reaction with $O$-phthaldialdehyde and $N$-acetyl-L-cysteine. First, as a Schlieren effect was observed, measurements were taken at $344 \mathrm{~nm}$ with respect to a baseline point at $700 \mathrm{~nm}$. On the other hand, as the reaction kinetics were strongly matrix dependent, the analytical readout should be evaluated at the final reaction equilibrium. In order to decrease the time spent in each determination, four parallel reaction coils were connected to different lateral ports of the selection valve. By use of this configuration, four samples were processed simultaneously. Grape juice samples were analysed $(n=15)$ and results were compared to a LC method. Except for two samples, only small deviations were found between the two methods. The developed method was also applied to monitoring two alcoholic fermentations (both white and red grape juices).

\section{Use of immobilised enzymes to enhance selectivity}

Reactions catalysed by enzymes are often used to provide selectivity for analytical methods when applied to complex samples. In SIA systems, enzymes can be used in solution [6] or immobilised in different materials [12,14,15]. Three different SIA systems for wine analysis using immobilised enzymes were described using different strategies to implement its catalytic action in-line. 
In the system proposed by Mayer and Ruzicka [14], the bead-injection concept [21] was utilised to prevent fouling of the immobilised enzymes for determination of glucose and ethanol. Using this approach, non-conducting beads containing immobilised glucose oxidase or alcohol oxidase were used to form renewable enzymatic layers adjacent to the surface of a platinum working electrode, where the current was continuously monitored. Hence, production of hydrogen peroxide from each analyte was detected during the passage of sample through the beads, depending on the enzyme chosen. After detection, the beads were discarded and a new enzymatic layer was formed in the next analytical cycle. Only one diluted white wine sample was tested and results were compared to the reference enzymatic procedures using commercial kits.

A SIA system containing an injection valve and an additional pump was described for the determination of ethanol and glycerol in wines [12]. In this case, reactors containing either alcohol dehydrogenase or glycerol dehydrogenase immobilised on controlled pore glass were placed in two loops connected to the injection valve. Using this configuration, the reaction product (NADH) obtained from one enzyme reactor was pumped towards the spectrophotometer while a plug of sample and co-factor was sent to fill the other enzyme reactor. This operational cycle enhanced sample throughput by alternating sample preparation and detection for the two determinations. Fifteen wine samples (red, white and Port) were analysed after dilution and the results were compared to those obtained by the UV enzymatic method (glycerol) or the hydrometric reference method (ethanol). No evidence of systematic differences between the two sets of results was found for either determination.

Another strategy to incorporate immobilised enzymes in SIA systems was through screen printed electrodes as described by Niculescu et al. [15]. In this case, quinohemoprotein alcohol dehydrogenase was immobilised in a redox polymer through cross-linking; the resulting membrane was placed on the electrode surface. This system was applied to determinations in three wine samples and the results were compared to the value declared by the producer. The analyser was also applied to monitoring model and industrial fermentation of grape juice; free cell sampling and sample dilution were performed in-line by means of an in-line filtration unit and a dilution chamber, respectively. The results obtained were in good agreement with those provided by the off-line determination using UV-enzymatic test kits.

\section{Multi-parameter systems}

One of the main advantages of SIA when compared to FIA is its flexibility and thus the possibility to accommodate different determinations without physical changes in the manifold configuration. In fact, determination of different analytes can be carried out using the same SIA manifold by changing the parameters introduced in the controlling software.
This approach was used in the determination of zinc, manganese, iron and copper using a SIA system with FAAS detection [18]. In this case, different sample volumes were chosen depending on the metal to be determined, as the linear response interval of the detection system was different for each of them. This was accomplished through software control, without physical reconfiguration of the system. The metals were determined in 20 samples (Port, Verde and Maduro Portuguese wines). These results were compared to those obtained by reference FAAS methods and good correlation was obtained for all analytes.

Different analytes can also be determined in the same SIA system by clustering different reagents in the lateral ports of the selection valve. In the SIA system for enzymatic determination of glucose and ethanol [14], the detection system response was targeted toward one of the analytes by choosing the bead suspension bearing the appropriate enzyme.

In SIA systems different determinations can also be accomplished during the same analytical cycle. This was achieved in the enzyme-based system described for determination of ethanol and glycerol [12] and in the FTIR system where multivariate analysis was implemented [20].

\section{Conclusions}

In the SIA systems described for wine analysis, parameters which are important for fermentation monitoring and wine stability assessment were evaluated. In several of these systems problems relating to matrix complexity were successfully avoided by implementing in-line sample treatment through different strategies. Hence, application to red wine samples was possible even when spectrophotometric detection was employed. Moreover, multi-parameter determination and adaptation to different types of wine were attained through software control, without changes in the manifold.

Although discussed in many articles, implementation of SIA systems in the wine industry for routine quality control was not reported. Nevertheless, fermentation monitoring was achieved for ethanol production [15] and amino acids consumption [11]. Direct sampling from reactors was attained for determination of ethanol [15]. This feature can easily be accomplished in SIA systems as its discontinuous flow mode enables in-line sample handling through filters and other preparation devices.

Not ignoring what was achieved by previous techniques, automation of wine analysis can still be a promising field for application of SIA. In our opinion, further investigation should be focused in the development of screening tools for contaminants (pesticides and herbicides) possibly by coupling SIA to chromatographic techniques. Although some work has been done, potentialities presented by SIA systems are far from being completely explored. 


\section{Acknowledgements}

The authors wish to thank Ministério da Agricultura, do Desenvolvimento Rural e das Pescas for financial support through project AGRO 273.

\section{References}

[1] A.S. Curvelo Garcia, J.C. Bouvier, Enologia 12 (1988) 27-43.

[2] A.M.R. Ferreira, J.L.F.C. Lima, T.I.M.S. Lopes, A.O.S.S. Rangel, J. Int. Sci. Vigne Vin. 27 (1993) 67-97.

[3] V. Cerdá, J.M. Estela, R. Forteza, A. Cladera, E. Becerra, P. Altimira, P. Sitjar, Talanta 50 (1999) 695-705.

[4] J. Ruzicka, G.D. Marshall, Anal. Chim. Acta 237 (1990) 329-343.

[5] C.E. Lenehan, N.W. Barnett, S.W. Lewis, Analyst 127 (2002) 9971020.

[6] A.N. Araújo, J.L.F.C. Lima, M.L.M.F.S. Saraiva, E.A.G. Zagatto, Am. J. Enol. Vitic. 48 (1997) 428-432.

[7] C.C. Oliveira, E.A.G. Zagatto, A.N. Araújo, J.L.F.C. Lima, Anal. Chim. Acta 371 (1998) 57-62.

[8] A.N. Araújo, J.L.F.C. Lima, A.O.S.S. Rangel, M.A. Segundo, Talanta 52 (2000) 59-66.
[9] J.Y. Neira, N. Reyes, J.A. Nóbrega, Lab. Rob. Autom. 12 (2000) 246-252.

[10] M.A. Segundo, A.O.S.S. Rangel, Anal. Chim. Acta 427 (2001) 279286.

[11] B. Muik, A. Edelmann, B. Lendl, M.J. Ayora-Cañada, Anal. Bioanal. Chem. 374 (2002) 167-172.

[12] M.A. Segundo, A.O.S.S. Rangel, Anal. Chim. Acta 458 (2002) 131138.

[13] H.R. Silva, M.A. Segundo, A.O.S.S. Rangel, J. Braz. Chem. Soc. 14 (2003) 59-64.

[14] M. Mayer, J. Ruzicka, Anal. Chem. 68 (1996) 3808-3814.

[15] M. Niculescu, T. Erichsen, V. Sukharev, Z. Kerenyi, E. Csöregi, W. Schuhmann, Anal. Chim. Acta 463 (2002) 39-51.

[16] G.C. Luca, B.F. Reis, E.A.G. Zagatto, M.C.B.S.M. Montenegro, A.N. Araújo, J.L.F.C. Lima, Anal. Chim. Acta 366 (1998) 193199.

[17] N. Zárate, A.N. Araújo, M.C.B.S.M. Montenegro, R. Pérez-Olmos, Am. J. Enol. Vitic. 54 (2003) 46-49.

[18] R.C.C. Costa, M.I. Cardoso, A.N. Araújo, Am. J. Enol. Vitic. 51 (2000) 131-136.

[19] R.C.C. Costa, A.N. Araújo, Anal. Chim. Acta 438 (2001) 227 233.

[20] R. Schindler, R. Vonach, B. Lendl, R. Kellner, Fresenius J. Anal. Chem. 362 (1998) 130-136.

[21] J. Ruzicka, L. Scampavia, Anal. Chem. 71 (1999) 257A-263A. 